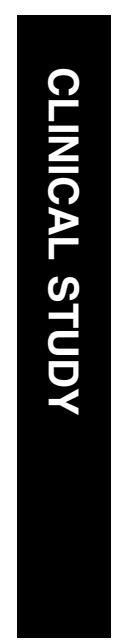

\title{
Poor agreement between Goldmann and Pascal tonometry in eyes with extreme pachymetry
}

\begin{abstract}
Aim To compare the intraocular pressure (IOP) measurements obtained using the Pascal dynamic contour tonometer (PDCT) with the standard Goldmann applanation tonometry (GAT) and to correlate them with the central corneal thickness (CCT) in a non-

glaucomatous population.

Methods We prospectively measured IOP using PDCT and GAT in random order in $\mathbf{1 0 0}$ normal eyes. CCT was analysed with an ultrasonic pachymeter in each case. Statistical analysis of baseline and stratified data included intraclass correlation coefficient (ICC), Lin correlation, and Bland-Altman analysis to evaluate the agreement between both techniques.

Results GAT was used first in 51 eyes and PDCT in 48 cases. Mean IOP was $14.8 \mathrm{~mm} \mathrm{Hg}$ with GAT and $20.3 \mathrm{~mm} \mathrm{Hg}$ with PDCT. Mean pachymetry was 553.23 micrometres $(\mu \mathrm{m})$ (SD: $4.7 \mu \mathrm{m})$. Global agreement of IOP between GAT and PDCT was 0.09 by ICC and 0.170 by Lin correlation. When CCT values ranged between 540 and $545 \mu \mathrm{m}$, the agreement between both tonometers was optimal (ICC 0.54 and Lin 0.61 ). Outside these pachymetry values, agreement between both tonometers diminished dramatically.

Conclusion Statistically significant agreement between both devices was reached only at intermediate pachymetry readings in contrast with other studies that show excellent global agreement between GAT and PDCT. Eye (2009) 23, 536-542; doi:10.1038/eye.2008.90; published online 28 March 2008
\end{abstract}

Keywords: Pascal tonometer; Goldmann tonometer; intraocular pressure; pachymetry; agreement; tonometry
E Milla' ${ }^{1}$ S Duch ${ }^{1}$, O Buchacra ${ }^{1}$ and C Masuet $^{2}$

Introduction

In a clinical scenario, 'safe' intraocular pressure (IOP) might be defined as pressure that does not lead to glaucomatous damage of the optic nerve head. Since the first tonometer designed by Von Graefe in 1862, the need for a reliable,

reproducible IOP measurement has originated the conception of multiple tonometers based on different physical principles. The Schiotz tonometer appeared in 1905 and was the prototype of indentation tonometry, which depended on corneal hysteresis and scleral rigidity. ${ }^{1}$ Goldmann applanation tonometry (GAT), introduced in 1954, was based on the Imbert-Fick law, which states that an external force $(\mathrm{W})$ against a sphere equals the pressure in the sphere $(\mathrm{P} 1)$ in relation to the area applanated by an external force (A): $W=P 1 \times A$. The applanation diameter chosen for GAT was $3.06 \mathrm{~mm}$ and the force of $1 / 10$ th gram over this area is equivalent to the pressure of a $1 \mathrm{~mm}$ high column of mercury. ${ }^{2}$

Since then, GAT has withstood the test of time and has remained the gold standard for IOP control. ${ }^{3}$ All current tonometers are calibrated and validated against it. The hand-applanation tonometers were found to be in satisfactory agreement with the GAT, whereas the Schiotz tonometer gave significantly low values in the pressure range considered. ${ }^{4}$ Many other tonometers have been tested and qualified as not accurate enough for clinical use, as they did not correlate well with GAT.

In 1975, Ehlers was the first author who pointed out the influence of pachymetry upon tonometry readings. ${ }^{5}$

Nowadays, it is known that one major limitation of GAT is its bias due to central corneal thickness (CCT) variation. The Goldmann tonometer calibration is based on an 
average CCT of $520 \mu \mathrm{m}$ and there is an underestimation or overestimation of IOP in thinner or thicker corneas, respectively. $^{5-8}$

Today, the influence of corneal structure upon IOP readings has gained renewed importance, especially after the trend towards LASIK surgery. For the first time in the history of glaucoma, the utility of GAT is being questioned as many studies have proved that this device underestimates IOP in LASIK-operated eyes. Other tonometers appear to be more useful in these eyes, such as the contact pneumotonometer. ${ }^{9}$

The Pascal dynamic contour tonometer (PDCT) uses a novel digital non-applanation contact tonometer to offer IOP readings free of any influence from corneal architecture, which has been extensively described in the paper by Kaufmann et al. ${ }^{10}$ This new tonometer possesses a concave tip that matches the corneal surface with a miniaturized pressure sensor in the middle. With this method, the contour surface is calculated to generate minimum distortion of the cornea and to direct all forces acting within the cornea to the pressure sensor surface. The IOP reading starts when the tip is properly positioned, thus limiting the degree of corneal deformation. Simultaneously, the tonometer provides a measurement of the ocular pulse amplitude.,

The majority of studies comparing GAT and PDCT show an excellent correlation between both devices, although Pascal readings tend to be slightly higher in general. Also, they all underline the lack of influence of corneal architecture upon Pascal tonometry. ${ }^{8,10-12}$

We undertook a prospective randomized study in a cohort of 100 normal patients whose IOP was evaluated by GAT and Pascal dynamic contour tonometry to study the agreement between both devices and the influence of corneal pachymetry upon the IOP readings.

\section{Methods}

A cohort of 100 consecutive non-glaucomatous normal individuals who attended our emergency department was recruited after a written consent was obtained in each case. All gave their informed consent to participate in the study, which adhered to the tenets of the Declaration of Helsinki. Only one normal eye from each patient was introduced in the study. They were all otherwise healthy, non-glaucomatous eyes. In this prospective, consecutive, comparative study undertaken in a clinical setting, patients were randomly divided into two groups:

\section{Group 1}

GAT was tested first with a Goldmann applanation tonometer (Haag Streit, Koeniz, Switzerland) and a mean of three consecutive readings was calculated.

Afterwards, these patients underwent PDCT with a Pascal tonometer (SMT Swiss Microtechnology AG, Zurich, Switzerland).

Only PDCT measures of q1 and q2 quality were included in the final analysis.

\section{Group 2}

Pascal tonometry was carried out first and immediately afterwards the three Goldmann tonometry readings were performed. The mean of the three readings was recorded.

Ultrasound pachymetry was performed in all cases after the tonometry procedures under topical anaesthesia with an Ocuscan RxP pachymeter (Alcon Laboratories Inc., Irvine, CA, USA) that provides six central readings and calculates the mean value.

All the IOP and pachymetry measurements were performed by the same observer.

\section{Statistical analysis}

For pooled analysis of paired measurements, patient data were aggregated for each IOP mode. The accuracy of the various IOP modes (average of three repeated measures) was determined by the agreement between the Goldmann and the Pascal tests globally and stratified by pachymetry.

(a) Using the method of differences, the Bland-Altman approach, ${ }^{13,14}$ which is a data scale assessment of agreement with the underlying model formulated as a two-way analysis of variance, the following were calculated:

(i) Mean difference, standard vs test, and 95\% limits of agreement.

(ii) Graphical display of difference vs mean, standard $v$ s test, not the difference against standard method. The plot was used to inspect whether the difference and its variance were constant as a function of the average, via the correlation of the difference $v$ s the average; a value near zero implied agreement.

(iii) The bias (mean difference) and precision (SD of the differences) between Goldmann and Pascal tests were calculated for pooled data by using the Bland-Altman approach.

(b) The concordance was studied by two coefficients, namely, the intraclass correlation coefficient (ICC) and the Lin concordance correlation:

(i) The ICC is a measure of reliability of a paired measure, so a measure reliability (ranging from 0 , no agreement, to +1 , perfect agreement) is defined as the ratio of the variance of interest to 
the total variance. The optimal levels of reliability have been suggested for an ICC of $0.7-0.75$.

(ii) Lin concordance correlation coefficient (Lin $\left.\mathrm{CCC}=\rho_{c}\right)^{15,16}$ was also calculated for each comparison. The Lin CCC, a parametric relationship-scale approach, compares agreement between two sets of measurement by assessing the variation from the 45-degree line through the origin, the line of perfect concordance $\left(\rho_{c}=1-\right.$; expected squared perpendicular deviation from 45-degree line/ expected squared perpendicular deviation from 45-degree line when $y$ and $x$ are uncorrelated). ${ }^{17}$ Thus, $\rho_{c}$ may be considered as a product of precision $(\rho)$ and a bias-correction factor, $C b$, a measure of accuracy, and $-1 \leqslant \rho_{c} \leqslant 1$.

The Pearson correlation coefficient $(r)$ was not determined as it is not a good agreement measure. In fact, the ' $r$ ' implies that data from two variables $(x, y)$ with perfect correlation $(r=1)$ lie on a straight line which, however, may not pass through the origin or have a slope equal to unity.

The ICC and the Lin CCC estimate the agreement in two ways: by the variance components procedure and by the moment method, ${ }^{18,19}$ with observers as fixed effects. Although the propriety of the ICC in agreement studies has been questioned, there is no consensus on this question.

The Lin and Bland-Altman approaches are complementary, indicating agreement (or lack of) on two different scales; although described only 3 years after the approach of Bland-Altman, Lin CCC is 'not very frequently used', but it has found recent endorsement. ${ }^{20}$

Finally, we have used a general linear model (GLM) of repeated measures to obtain prediction models of IOP by the Pascal test as a paired measurement of the IOP measured by Goldmann. Effect sizes were calculated using partial eta square $\left(\eta_{p}^{2}\right)$, which estimates the degree of association in the sample. Collinearity was studied by tolerance values being considered tolerance values of which values $<0.20$ indicated excessive collinearity.
All statistical analyses were conducted using SPSS for Windows version 12 (SPSS Inc., Chicago, IL, USA) and MedCalc for Windows version 9.2 (MedCalc Software, Mariakerke, Belgium). For all tests, a $P<0.05$ was considered to be statistically significant.

We certify that all applicable institutional and governmental regulations concerning the ethical use of human volunteers were followed during this research.

\section{Results}

A total of 99 eyes were included in the final analysis. GAT was used first in 51 eyes and PDCT in 48. Mean IOP was $14.8 \mathrm{~mm} \mathrm{Hg}$ with GAT and 20.3 with PDCT.

Mean pachymetry was $553.23 \mu \mathrm{m}$ (SD: $36.5 \mu \mathrm{m}$; range $237 \mu \mathrm{m}$; minimum $452 \mu \mathrm{m}$; maximum $689 \mu \mathrm{m}$ ).

Table 1 lists IOP measurements with both instruments as well as CCT in both groups, and Figure 1 displays IOP readings with both methods considering the pachymetry values.

Coefficients of concordance between both methods are expressed in Table 2 by two different statistical equations, namely the ICC and the Lin correlation coefficient.

The bias (mean difference) between the pooled Goldmann and Pascal-tests (Figure 2) was $-5.43 \mathrm{mmHg}$. The precision (SD) of the pooled differences was 0.48. Using these figures, the $95 \%$ limits of agreement (ie, bias \pm 1.96 ) between the Goldmann and Pascal tests were $-5.43 \pm 4.76$.

The findings indicate that the $95 \%$ limits of agreement (bias $\pm 1.96 \mathrm{SD}$ ) were -5.43 (95\% CI: $-14.8 / 3.9$ ). In other words, $95 \%$ of all pooled Pascal measurements were within a range from -14.8 to $3.9 \mathrm{~mm} \mathrm{Hg}$ from Goldmann measurements. A systematic bias was not observed, as data were dispersed around the mean, not being statistically significant in the presence of pooled data above or below the mean difference between the two studied tests.

This bias presents an even wider range depending on pachymetry (Figure 3 ) not even statistically significant $(P=0.380)$ (ie, bias -3.41 (SD 1.75) in pachymetry $540-545 \mu \mathrm{m}$, and bias -8.05 (SD 4.88) in pachymetry $<513 \mu \mathrm{m})$.

Table 1 IOP measurements with both instruments as well as central corneal thickness (CCT)

\begin{tabular}{lllcccccc}
\hline Tonometer & Order & $\mathrm{N}$ & Mean IOP $(\mathrm{mm} \mathrm{Hg})$ & $S D$ & $\mathrm{P}$ & CCT $(\mu m)$ & SD $(\mu m)$ & Tip. error \\
\hline GAT $^{\mathrm{a}}$ & First & 51 & 16.098 & 3.36 & 0.00 & 561.705 & 37.873 & 5.3 \\
& Second & 48 & 13.604 & 2.84 & 0.00 & & 3.93 & 4.7 \\
PDCT & First & 48 & 20.139 & 4.75 & 0.00 & 544.229 & 32.937 & \\
& Second & 51 & 20.505 & 4.75 & 0.00 & & & \\
\hline
\end{tabular}

${ }^{\mathrm{a}}$ Mean of three consecutive GAT readings. $\mathrm{SD}=$ standard deviation; $\mathrm{CCT}=$ central corneal thickness. 


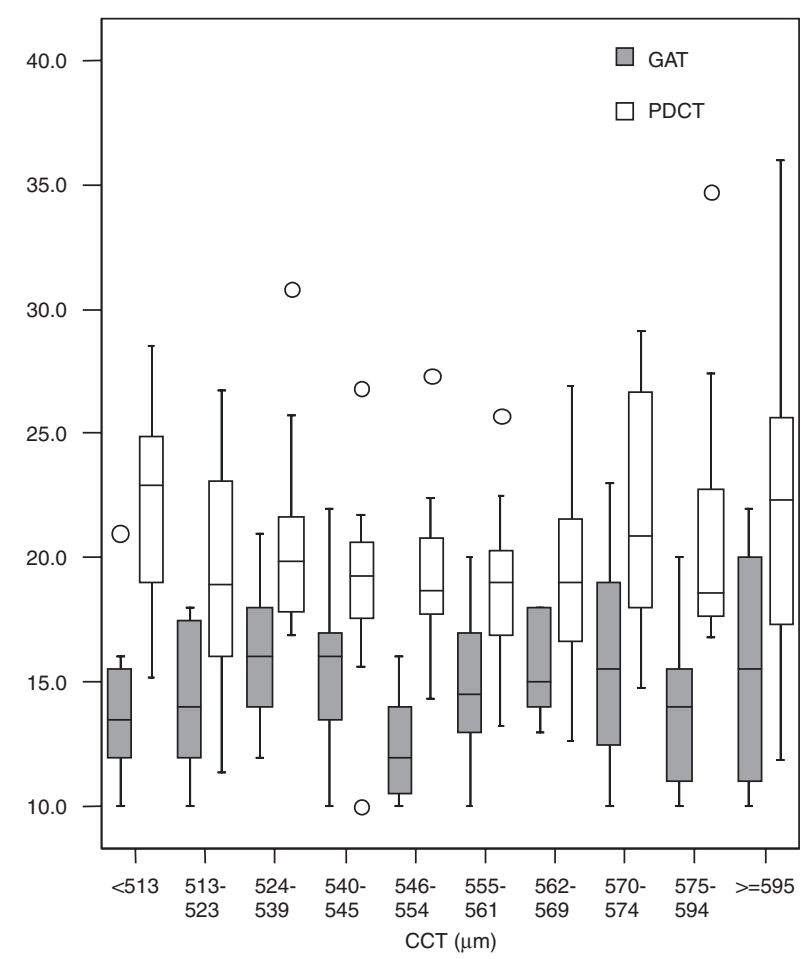

Figure 1 Description of IOP by Pascal and Goldmann test considering the pachymetry (CCT).

Table 2 Description of global and specific agreement between GAT and PDCT depending on central corneal thickness (CCT) evaluated by ICC and Lin correlation coefficient

\begin{tabular}{lrrrc}
\hline CCT & $\mathrm{N}$ & \multicolumn{1}{c}{ ICC } & P-value & Lin CC (CI 95\%) \\
\hline General & & -0.08556 & 0.001 & $0.170(0.070-0.266)$ \\
$<513$ & 8 & -0.46810 & 0.002 & $0.056(-0.168-0.275)$ \\
$513-523$ & 11 & -0.07595 & 0.007 & $0.162(-0.154-0.449)$ \\
$524-539$ & 10 & -0.19820 & 0.011 & $0.090(-0.230-0.393)$ \\
$540-545$ & 7 & $\mathbf{0 . 5 3 7 6 6}$ & $<\mathbf{0 . 0 0 1}$ & $\mathbf{0 . 6 1 0}(\mathbf{0 . 2 9 7 - 0 . 8 0 5 )}$ \\
$546-554$ & 12 & -0.61504 & 0.004 & $-0.020(-0.206-0.167)$ \\
$555-561$ & 10 & -0.14102 & 0.017 & $0.108(-0.277-0.463)$ \\
$562-569$ & 11 & -0.04009 & 0.011 & $0.172(-0.141-0.454)$ \\
$570-574$ & 8 & 0.08487 & 0.006 & $0.303(-0.115-0.629)$ \\
$575-594$ & 12 & -0.12237 & $<0.001$ & $0.207(-0.021-0.415)$ \\
$\geqslant 595$ & 10 & -0.12257 & 0.032 & $0.090(-0.307-0.461)$
\end{tabular}

Bold values signify optimal agreement between both tonometers.

The agreement between the IOP measured using the ICC between the Pascal and the Goldmann tests was 0.09 and 0.170 according to the Lin CCC.

So, globally, the agreement between both tests is moderate, with a marked tendency of PDCT to overestimate IOP, as we could see by the Bland-Altman approach.

In patients with pachymetry values between 540 and $545 \mu \mathrm{m}$, the agreement is the optimal between both tonometers (ICC 0.54 and Lin 0.61).

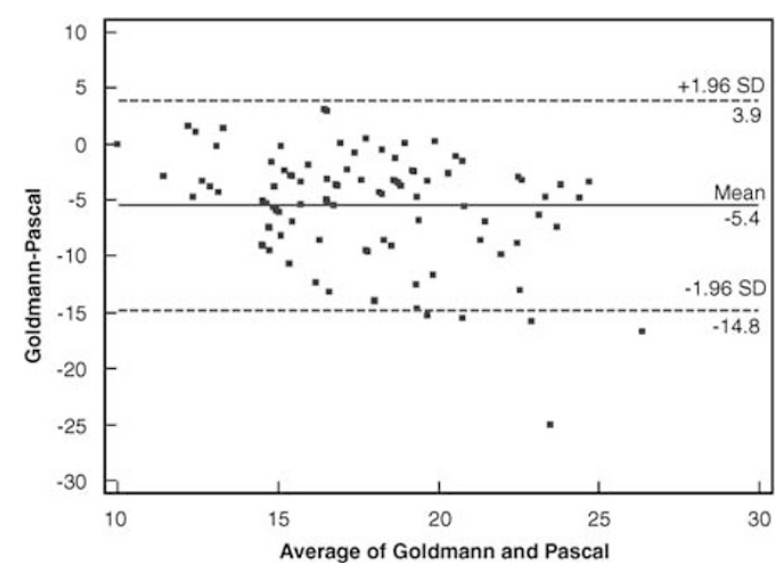

Figure 2 Bias and precision of Pascal compared with Goldmann test using a Bland-Altman approach globally.

The GLM repeated measures showed a significant main effect for IOP by the Pascal test $\left(F_{(3,95)}=4.956\right.$; $P=0.003 ; \eta_{p}^{2}=0.127$ ) with a low collinearity (tolerance values $>0.940$ ). Although the GLM showed that means between the Pascal and Goldmann tests were significantly different $(P=0.003)$, the effect size was small to modest $\left(\eta_{p}^{2}=0.127\right)$.

\section{Discussion}

Accurate measurement of IOP is a fundamental parameter during eye examination and glaucoma evaluation. Over the past four decades, GAT has become the standard for routine measurement of IOP, as the method has proven to be robust and easy to use with low intra- and interobserver variability. ${ }^{3}$ However, the accuracy of GAT depends on many factors, including corneal thickness, corneal curvature, and corneal structure. Using a cornea biomechanical model, $\mathrm{Liu}^{21}$ demonstrated that differences in corneal biomechanics across individuals may have greater impact on IOP measurement errors than corneal thickness or curvature.

It is known that GAT was calibrated for a CCT of 520 micrometres, and therefore it underestimates IOP in eyes with thin corneas and overestimates IOP in eyes with thick corneas. ${ }^{5-8}$ There are also other sources of error such as hypo/hyperfluorescence of the precorneal tear film, accommodation, the Valsalva manoeuvre, vertical gaze and variations in the corneal resistance to indentation among others. ${ }^{22}$

However, 'normal' CCT varies when different pachymeters and populations are considered. Using optical pachymeters, CCT tends to be thinner compared with the readings given by ultrasonic devices. ${ }^{23}$

Besides, these measurements require a reliable nomogram to convert GAT readings into true IOP. 


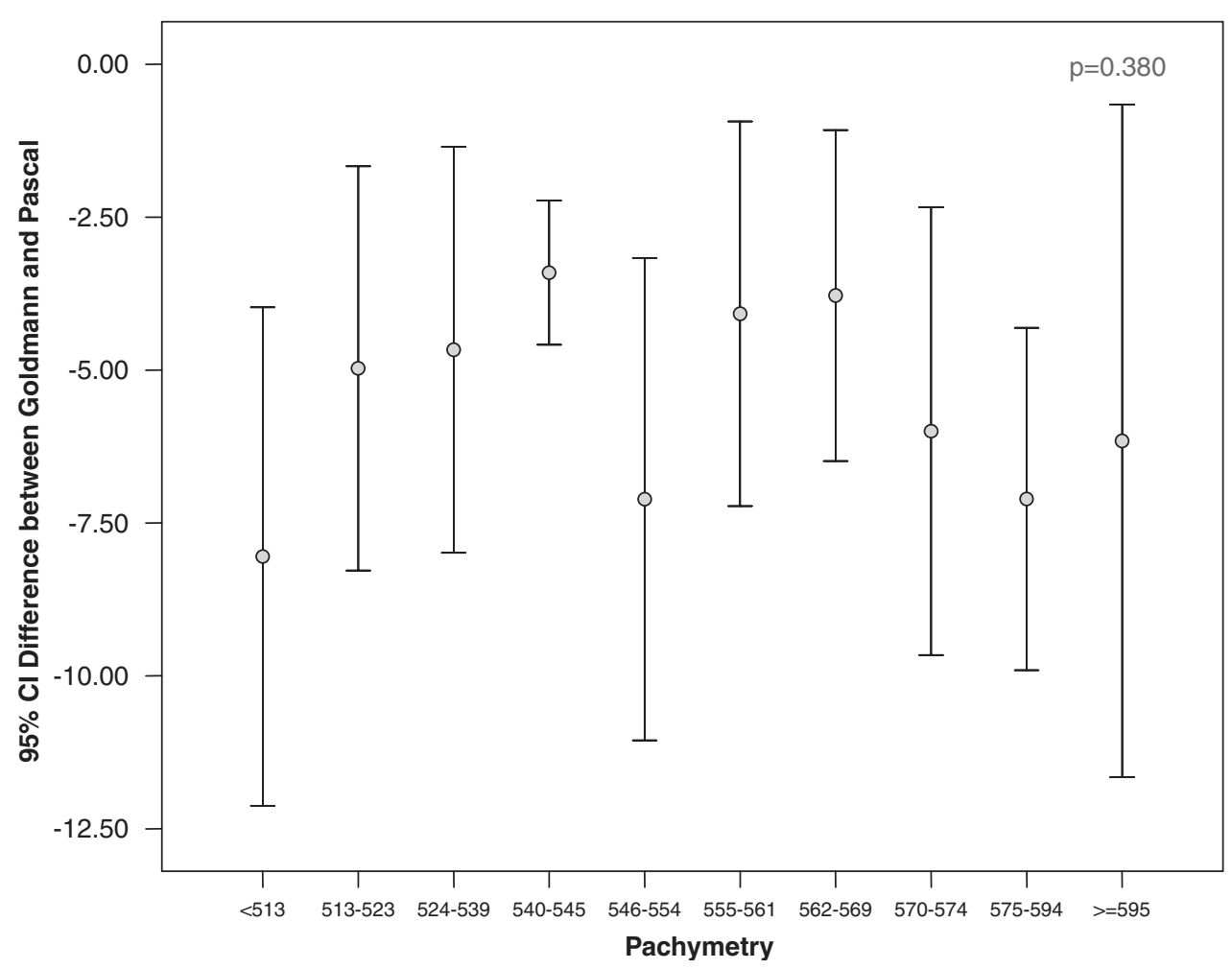

Figure 3 Bias and precision of Pascal compared with Goldmann test using a Bland-Altman approach depending on the pachymetry measures.

Several nomograms for adjusting GAT readings in normal eyes with varying CCT or in eyes after refractive surgery have been published as the Ehlers formula and the Orssengo-Pye model, ${ }^{6,24}$ but so far, none seems to be satisfactory. These models could be erroneous and lead to an overcorrection of IOP, thus resulting in erroneously low corrected IOP eyes with thicker cornea and erroneously high corrected IOP in eyes with thinner cornea. ${ }^{25}$ According to the meta-analysis performed by Doughty that included 600 sets of CCT data, the impact of CCT on applanation tonometry of healthy eyes is unlikely to achieve clinical significance, but for corneas of eyes with chronic disease, pachymetry should be performed if the tonometry reveals IOP readings that are borderline or unusual, and the correction for these eyes should be 2 or $3 \mathrm{~mm} \mathrm{Hg}$ for a $0.05 \mathrm{~mm}$ difference in CCT from $0.535 \mathrm{~mm} .^{26}$

Looking for the most accurate IOP measurement, GAT has been compared with many other tonometers. Three tonometers (Tono-Pen, Schiotz, and the Pro-Ton) showed a small significant underestimation of the Goldmann IOPs, ${ }^{27}$ and contact pneumotonometry was more reliable when measuring eyes where laser in situ keratomileusis for myopia was performed. ${ }^{9}$

PDCT is a novel device designed for IOP measurements and it is assumed to be largely independent of the corneal architecture. Its tip adapts to the corneal surface causing minimal distortion of this structure in contrast to what happens with GAT.

Several published studies have demonstrated good correlation between both tonometers, although PDCT readings are sensibly and consistently higher owing to the fact that PDCT was calibrated against a manometrically controlled pressure standard rather than a GAT pressure reading. ${ }^{10}$ Several studies include normal eyes, ${ }^{10,11}$ whereas others are performed in eyes with glaucoma ${ }^{7,8,12}$ and therefore their results cannot be matched.

Pache et $a l^{11}$ found that in normal eyes, PDCT allows suitable and reliable IOP measurements in good concordance with GAT, and none of the tonometers showed a significant correlation with CCT, except for a mild increase of the difference (GAT-PDCT) in thicker corneas. In this study, mean PDCT readings were, on average, $1 \mathrm{~mm} \mathrm{Hg}$ higher than GAT values. Similarly, Kaufmann et $a l^{10}$ studied 228 healthy eyes in which high concordance between the same both tonometers was observed, and thus the IOP PDCT readings were slightly higher. Doyle and Lachkar ${ }^{28}$ reported that the mean GATPDCT difference was $-2.6 \mathrm{~mm} \mathrm{Hg}$ in thin corneas and $-0.06 \mathrm{~mm} \mathrm{Hg}$ in thick corneas; therefore, their conclusion is that in structurally normal thin corneas, PDCT may 
give a more accurate assessment of the true IOP, but it does not appear to have any benefit over GAT in thick corneas. The study by Salvetat et $a l^{7}$ in glaucomatous eyes showed that PDCT measurements were $3.2 \mathrm{~mm} \mathrm{Hg}$ higher than GAT readings; the authors found a fair intermethod agreement and lack of correlation with CCT. $\mathrm{Ku}$ et $\mathrm{al}^{8}$ also studied glaucoma eyes and found that PDCT readings $2.0 \pm 2.1 \mathrm{~mm} \mathrm{Hg}$ were higher than those of GAT; they found a significant linear relationship between both devices, except in thicker corneas in which the agreement between PDCT-GAT was poorer than in intermediate or thinner corneas. This is in concordance with Detry-Morel et $a l^{12}$ who recently found that IOP measurements with the PDCT and GAT correlated well and were reproducible. PDCT IOP measurement variability was slightly higher than GAT with relatively wide $95 \%$ limits of agreement. Considering the entire study population (normal, glaucoma, and ocular hypertensive individuals), PDCT overestimated IOP by a mean $2.0 \mathrm{~mm} \mathrm{Hg}$ compared with GAT. According to these authors, PDCT was independent of CCT, especially in thin corneas, but did not appear to be clinically advantageous over the GAT in the IOP measurement in thick corneas.

The IOP measurements obtained by GAT and PDCT demonstrate a high concordance between the two techniques, according to the above mentioned studies $(r>0.5)$, even though IOP readings obtained by PDCT are consistently higher $(0.8-4 \mathrm{~mm} \mathrm{Hg})$.

In our study, IOP readings obtained by PDCT were $5.5 \mathrm{~mm} \mathrm{Hg}$ higher on average than the readings obtained by GAT. This difference became even larger in cases in which IOP was measured by GAT with a prior PDCT reading. We could observe that the Pascal readings were also higher when grouped by pachymetry values. These differences become even higher with increasing IOP values as shown in Figure 1.

All results were strongly influenced by CCT, as concordance between both tonometers was optimal only at intermediate pachymetry values. So, globally, the agreement between both tests is moderate, with a marked tendency of PDCT to overestimate IOP.

In patients with pachymetry values between 540 and $545 \mu \mathrm{m}$, the agreement was the optimal between both tonometers, as it was demonstrated by two different statistical coefficients. Interestingly, these CCT average eyes are the ones with less influence upon GAT IOP readings. On the other hand, lack of concordance in the rest of the CCT measurements was also demonstrated by those two statistical methods.

We can conclude that in our study, there was a lack of correlation between GAT and PDCT either in thinner or thicker corneas. The difference in CCT between the two groups (GAT used first and PDCT used first) was not statistically significant. Global correlation between both tonometers also showed a lack of significance.

Finally, we have created a prediction model of IOP by the Pascal test considering the Goldmann measure (the Gold Standard), the pachymetry, and sex of patients using a GLM of repeated measures. We have observed that there is sufficient evidence to affirm that the Pascal measurements and Goldmann measurements are not statistically equal $(P=0.003)$ and that the variability is explained only to a lesser extent by sex, pachymetry, and Goldmann measures $\left(R^{2}=0.135\right.$; and $\left.\eta_{p}^{2}=0.127\right)$. Future research comparing different tests in IOP should be undertaken to obtain further evidence for this assumption. These results differ from several published studies in which IOP measurements taken with PDCT showed an excellent global agreement with the ones obtained by GAT.

We believe that CCT is an essential variable to consider in interpreting IOP readings, especially for the GAT measurements. Demonstration of the relative independence of PDCT IOP measurements from CCT supports a potential clinical role for the PDCT, particularly for subjects with corneal pachymetry outside of the normal range.

However, the clinical implications of this fact upon glaucomatous optic neuropathy and its progression is still unclear. Since then, the pathogenic mechanisms of glaucoma are inevitably related to somehow 'uncertain' IOP values.

\section{Acknowledgements}

The results of this study were presented at the ARVO meeting in Fort Lauderdale, 2006.

\section{References}

1 Ravin JG, Higginbotham EJ. Are we there yet? Celebrating the centennial of the Schiotz tonometer. Arch Ophthalmol 2006; 124: 1337-1338.

2 Stuckey GC. Application of physical principles in the development of tonometry. Clin and Exp Ophthalmol 2004; 32: 633-636.

3 Wessels IF, Oh Y. Tonometer utilization, accuracy and calibration under field conditions. Arch Ophthalmol 1990; 108: 1709-1712.

4 Krieglstein GK, Waller WK. Goldmann applanation vs hand-applanation and Schiotz indentation tonometry. Albrecht Von Graefes Arch Klin Exp Ophthalmol 1975; 194: 11-16.

5 Ehlers N, Bramsen T, Sperling S. Applanation tonometry and central corneal thickness. Acta Ophthalmol 1975; 53: 34-43.

6 Johnson M, Kass MA, Moses RA, Grodzki WJ. Increased corneal thickness simulating elevated intraocular pressure. Arch Ophthalmol 1978; 96: 664-665. 
7 Salvetat ML, Zeppieri M, Tosoni C, Brusini P. Comparisons between Pascal dynamic contour tonometry, the TonoPen, and Goldmann applanation tonometry in patients with glaucoma. Acta Ophthalmol Scand 2007; 85: 272-279.

8 Ku JYF, Danesh-Meyer HV, Craig JP, Gamble GD, McGhee CNJ. Comparison of intraocular pressure measured by Pascal dynamic contour tonometry and Goldmann applanation tonometry. Eye 2006; 20: 191-198.

9 Duch S, Serra A, Castanera J, Abos R, Quintana M. Tonometry after laser in situ keratomileusis treatment. J Glaucoma 2001; 10: 261-265.

10 Kaufmann C, Bachmann LM, Thiel MA. Comparison of dynamic contour tonometry with goldmann applanation tonometry. Invest Ophthalmol Vis Sci 2004; 45: 3118-3121.

11 Pache M, Wilmsmeyer S, Lautebach S, Funk J. Dynamic contour tonometry vs Goldmann applanation tonometry: a comparative study. Graefes Arch Clin Exp Ophthalmol 2005; 243: 763-767.

12 Detry-Morel M, Jamart J, Detry MB, Ledoux A, Pourjavan S Clinical evaluation of the Pascal dynamic contour tonometer. J Fr Ophtalmol 2007; 30: 260-270.

13 Bland JM, Altman DG. Statistical methods for assessing agreement between two methods of clinical measurement. Lancet 1986; 1: 307-310.

14 Bland JM, Altman DG. Comparing methods of measurement: why plotting difference against standard method is misleading. Lancet 1995; 346: 1085-1087.

15 Lin LI. A concordance correlation coefficient to evaluate reproducibility. Biometrics 1989; 45: 255-268.

16 Lin LI, Hedayat AS, Yang M. Statistical methods in assessing agreement: models, issues and tools. J Am Stat Assoc 2002; 97: 257-270.

17 Dunn G. Statistical Evaluation of Measurement Errors: Design and Analysis of Reliability Studies. Hodder Arnold: London, 2004.
18 Carrasco JL, Jover L. Estimating the generalized concordance correlation coefficient through variance components. Biometrics 2003; 59: 849-858.

19 Carstensen B. Comparing and predicting between several methods of measurement. Biostatistics 2004; 5: 399-413.

20 Sanchez MM, Binkowitz BS. Guidelines for measurement validation in clinical trial design. J Biopharm Stat 1999; 9: 417-438.

21 Liu J, Roberts CJ. Influence of corneal biomechanical properties on intraocular pressure measurement: quantitative analysis. J Cataract Refract Surg 2005; 31: 146-155.

22 Whitacre MM, Stein R. Sources of error with use of Goldmann-type tonometers. Surv Ophthalmol 1993; 38: 1-30.

23 Li EY, Mohamed S, Leung CK, Rao SK, Cheng AC, Cheung CY et al. Agreement among 3 methods to measure corneal thickness: ultrasound pachymetry, orbscan ii, and visante anterior segment optical coherence tomography. Ophthalmology 2007; 114(10): 1842-1847.

24 Kirstein EM, Hüsler A. Evaluation of the Orssengo-Pye IOP corrective algorithm in LASIK patients with thick corneas. Optometry 2005; 76: 536-543.

25 Gunvant P, O'Leary DJ, Baskaran M, Broadway DC, Watkins RJ, Vijaya L. Evaluation of tonometric correction factors. J Glaucoma 2005; 14: 337-343.

26 Doughty MJ, Zaman ML. Human corneal thickness and its impact on intraocular pressure measures: a review and meta-analysis approach. Surv Ophthalmol 2000; 44: 367-408.

27 Wingert TA, Bassi CJ, Mac Alister WH, Galanis JC. Clinical evaluation of five portable tonometers. J Am Optom Assoc 1995; 66: 670-674.

28 Doyle A, Lachkar Y. Comparison of dynamic contour tonometry with goldman applanation tonometry over a wide range of central corneal thickness. J Glaucoma 2005; 14: 288-292. 